\title{
La cristalización del management industrial: historia desde la metateoría kuhniana*
}

The Crystallization of Industrial Management: History from the Kuhnian Metatheory

Leandro Ariel Giri ${ }^{\dagger \neq}$

\begin{abstract}
Resumen
En el presente artículo se presenta una reconstrucción del episodio histórico de la cristalización del management industrial a través de la introducción de la Teoría de la Dinámica de Sistemas de Jay Wright Forrester de la Sloan School of Management del MIT. Para ello, se utilizarán explícitamente las herramientas historiográficas provistos por el marco metateórico de Thomas Kuhn, por considerarlo de gran fertilidad para dar cuenta de los episodios de maduración de disciplinas científicas.
\end{abstract}

Palabras clave: management - Jay Wright Forrester - dinámica de sistemas - Thomas Kuhn

\begin{abstract}
In the present article we will present a reconstruction of the historical episode of the crystallization of industrial management through the introduction of Jay Wright Forrester's Systems Dynamics Theory from the MIT's Sloan School of Management. In order to achieve this, we will use historiographic tools explicitly provided by Thomas Kuhn's metatheoretical framework, as we consider it especially fruitful to give account of episodes of maturation of scientific disciplines.
\end{abstract}

Keywords: management - Jay Wright Forrester - systems dynamics - Thomas Kuhn

* Recibido: 10 de diciembre de 2020. Aceptado con revisiones: 15 de marzo de 2021.

† Instituto de Investigaciones Filosóficas (IIF-SADAF-CONICET)/Universidad Nacional de Tres de Febrero (UNTREF). Para contactar al autor, por favor, escribir a: leandrogiri@sadaf.org.ar.

${ }^{\sharp}$ Una versión muy preliminar de este trabajo fue comentada por Lucía Federico, Hernán Miguel, Pablo Melogno y Paul Hoyningen-Huene. A elles, mi agradecimiento.

Metatheoria 11(2)(2021): 1-15. ISSN 1853-2322. eISSN 1853-2330.

(C) Editorial de la Universidad Nacional de Tres de Febrero.

(C) Editorial de la Universidad Nacional de Quilmes.

Publicado en la República Argentina. 


\section{Introducción}

Existe una plétora de posibilidades para realizar una reconstrucción histórica, cada una con diversas ventajas y desventajas, con distintas potencialidades para enfatizar o disminuir la importancia de hechos e interpretaciones varias.

La sucesión de hechos históricos relevantes reseñados y ordenados cronológicamente no bastaría ante un panorama semejante. El famoso axioma de Ranke queriendo dar cuenta de "wie es eigentlich gewesen" (lo que realmente sucedió) llevaría a un escenario donde un conjunto de eventos en el espacio-tiempo pulula solitario en espera del demiurgo analítico que les otorgue un sentido. Se intentará aquí tomar el rol de dicha entidad metafísica, esperando conseguir una interpretación integral y sólida de lo que las fuentes historiográficas parecen mostrar. Es evidente la potencial dificultad metodológica que podría arrojar la adopción de una u otra herramienta filosófica de análisis. Pero al mismo tiempo son destacables las palabras de C. Ulises Moulines: "Un abordaje puramente narrativo de la historia de la ciencia sin compromiso metateórico es una visión tan empobrecida de ella como la idea de una ciencia de la naturaleza 'puramente empírica' sin influencia de una teoría” (Moulines 1983, p. 286). Sin embargo, lo más común es que los historiadores de la ciencia no revelen sus compromisos metateóricos, y por ello el lector los tenga que deducir de la lectura de los trabajos. Se evitará esta tediosa situación siendo explícitos al respecto, tomando una posición acorde al objetivo historiográfico perseguido. De todas maneras, la reconstrucción será acompañada de los detalles históricos biográficos y contextuales que permitirán entender que ningún elemento teórico es huérfano de su tiempo.

Pues bien, la historia del episodio de la evolución del management industrial que se quiere mostrar en el desarrollo de este trabajo no busca enfatizar procesos de observación, conjeturación y/o refutación como lo hacen algunos marcos como el Popperiano. Lo que se quiere mostrar en este caso es el proceso de cristalización ${ }^{1}$ del management industrial a través de un cambio conceptual en la disciplina general que lo llevó a asemejarse a una rama de la ingeniería, uniendo la casuística dispersa en un marco sistemático, en búsqueda de una madurez definitiva (que finalmente no se logró). Ante este panorama, se ha seleccionado el marco metateórico desarrollado por el físico, historiador y filósofo de la ciencia Thomas Kuhn para el tratamiento de la evolución conceptual de esta rama de la ciencia del management, dada la compatibilidad de los objetivos buscados con el marco historiográfico de su creación (ver p.e. Kuhn 1971, 2017, Giri \& Giri 2020). Se procederá entonces a reconstruir los elementos biográficos de Jay Forrester, introductor del cambio conceptual mencionado, y luego se compilarán aquellos elementos que permitirán la reconstrucción racional del episodio histórico en clave kuhniana.

El episodio histórico aquí presentado es el del proceso de consolidación de la Dinámica de Sistemas. de Jay Wright Forrester (p.e. 2013, 1968). Esta matriz disciplinar utiliza la idea de sistema abierto de retroalimentación (open-feedback systems), ubicua ya en la década de 1950 en diferentes ramas de la ingeniería, para dar cuenta de sistemas sociales complejos, en particular organizaciones industriales (aunque luego se ampliaría, como veremos, a otros dominios de aplicación) La Dinámica de Sistemas plantea entonces, mediante una analogía entre el proceso de gerenciamiento de una organización y el proceso de automatización de una máquina, una metodología de modelización que permite descripciones precisas del comportamiento organizacional y la posibilidad de probar el resultado de decisiones gerenciales in silico previo a la implementación en las organizaciones reales. La Dinámica de

\footnotetext{
${ }^{1}$ El sentido de "cristalización" al que hacemos referencia es informal, refiriéndose a un proceso de unificación de elementos teóricos previamente dispersos en diversas escuelas en competencia. Una noción mucho más precisa del término ha sido desarrollada por parte de C. Ulises Moulines (p.e. ver 2011), pero tal nivel de refinamiento excede el propósito de este artículo.
} 
Sistemas se fundamenta sobre todo en la Teoría de Sistemas de Información y Retroalimentación de Brown y Campbell (1948), aunque surge en una época donde el abordaje sistémico y la cibernética se hallan en ebullición y proliferan los avances en estos campos. Veremos los detalles de la matriz disciplinar en cuestión más adelante en el trabajo.

\section{Algunos elementos biográficos pertinentes}

Jay Wright Forrester nació en 1918 en una granja en Nebraska, en el seno de una familia culta. El negocio agrario, según Forrester (1989), constituyó el ejemplar paradigmático que fijó en su mente el modo de operar de los sistemas complejos, lo cual lo llevaría posteriormente a su Dinámica de Sistemas.

En su adolescencia, Forrester diseñó una pequeña planta eólica para su granja. Su pasión por la tecnología lo llevó a rechazar una beca de estudios en agricultura, para dirigirse a la Universidad de Nebraska con el fin de estudiar Ingeniería Eléctrica.

Luego de obtener su título de grado, en 1939, Forrester se dirigió al Massachussets Institute of Technology (MIT) para trabajar como asistente de investigación de Gordon Brown, pionero en el área de Servomecanismos. Del trabajo con Brown surgieron invenciones tecnológicas como un control de radar que operó en la Segunda Guerra Mundial, y que Forrester reparó mientras el buque que lo portaba, USS Lexington, era violentamente torpedeado por la marina japonesa (Forrester 1992).

Al final de la Guerra, Forrester decidió continuar trabajando en el MIT, donde se embarcó en el diseño y fabricación de un simulador de vuelo. Durante el desarrollo de éste, notó que el computador analógico de uso estándar en la época se hallaba limitado en su capacidad de procesamiento para poder simular correctamente el complejo entorno real de un vuelo.

Las computadoras de tipo analógico, como las utilizadas en los analizadores de redes del sistema de potencia eléctrica y en los analizadores de ecuaciones diferenciales, han sido desarrollados desde 1930 a 1950. [...] Sin embargo, estas computadoras demostraron ser inadecuadas para lidiar con problemas de interés práctico. No pueden lidiar efectivamente con sistemas no-lineales. (Forrester 2013, pp. 18-19)

Por esta razón, Forrester se avocó a la investigación en el área de computadoras digitales. En 1951 fundó el departamento de Computadoras Digitales en el MIT, donde dirigió el proyecto de desarrollo de la computadora Whirlwind, la cual fue utilizada para multiplicidad de aplicaciones (Lane \& Sterman 2011).

A pesar del éxito de Forrester como pionero de la computación digital, su camino personal adquirió un rumbo distinto al tomar, en 1956, un puesto de investigación en la Sloan School of Management del MIT. Este instituto había sido creado con el fin de crear un pensamiento nuevo en administración de base ingenieril, en contraposición al ambiente "blando" de las principales universidades de esa disciplina en EE.UU. (Harvard, Columbia y Chicago, ver Kochan \& Schmalensee 2003).

Un trabajo realizado por Forrester en 1956 en el ámbito de la planta generadora de electricidad de Kentucky de la General Electric, lo llevó a utilizar herramientas ingenieriles para diagnosticar y solucionar un problema administrativo en la compañía (básicamente, un trastorno organizativo debido a la fluctuación en la demanda de electricidad durante los meses de verano que llevaban a contratar personal que luego era superfluo, ver Forrester 1995). Ese episodio fue la inspiración para la Dinámica de Sistemas, la cual constituirá, como relataremos a continuación, la cristalización del management industrial moderno.

\section{La Dinámica de Sistemas}

La disciplina del management clásica se hallaba en un punto crucial de su historia en la década de 1950. Autores clásicos como Taylor (1919) y Weber (1924) ya habían declarado al management como una ciencia; Gulick y Urwick (1937) por su parte habían fijado los principios de la buena administración, 
aunque en 1947 Herbert Simon (1964), utilizando herramientas metateóricas heredadas del positivismo lógico refutaría tales principios y precisaría enormemente el alcance y competencia de los científicos de la administración, fusionando de alguna manera los principios matemáticos de las teorías de juego de Von Neumann y Morgenstern (1990) con las ideas organizacionales de Chester Barnard (1938) y modelos matemáticos del comportamiento. A pesar de ello, otros autores insistían en que aún no existían elementos para tratar al management como una ciencia (ver p.e. Drucker 1954, Gordon \& Howell 1959). Para muchos, entre ellos el protagonista de nuestro relato, el management de las décadas 1940 y 1950 era más un "arte" que una ciencia, y necesitaba renovarse con urgencia, especialmente su vertiente relacionada a la administración de organizaciones industriales: si bien existía cada vez más literatura específica al respecto, la misma consistía sobre todo en modelos matemáticos fácilmente resolubles y exactos, de escasa potencialidad para explicar sistemas empíricos reales. Para comprender el punto de vista de Jay Forrester a este respecto, se citará in extenso:

Todo empeño humano que valga la pena emerge primero como un arte. Tenemos éxito antes de que comprendamos por qué. La práctica de la medicina o de la ingeniería comenzó como un arte empírico representando solo el ejercicio del juicio basado en la experiencia. El desarrollo de las ciencias subyacentes fue motivado por la necesidad de entender mejor el fundamento en el cual el arte descansa. [...]

[...] El arte se desarrolla a través de la experiencia empírica, pero en el tiempo cesa de crecer debido al estado desorganizado de su conocimiento. Cuando la necesidad y las fundaciones necesarias coinciden, una ciencia se desarrolla para explicar, organizar y destilar la experiencia en una forma más compacta y utilizable. Mientras la ciencia crece, provee un nuevo fundamento para la expansión futura del arte.

Por siglos, el management como un arte ha progresado por la adquisición y almacenamiento de la experiencia humana. Pero mientras no haya una base científica ordenada subyacente, las experiencias permanecen como casos especiales. Las lecciones son pobremente transferibles ya sea en el tiempo o en el espacio. (Forrester 2013, pp. 1-2)

Que la literatura de la disciplina no poseyera más que casuística desorganizada tenía consecuencias severas:

El manager corporativo hoy encuentra poca ayuda en las experiencias almacenadas en la literatura desde hace una generación atrás. Las descripciones son incompletas y carecen de precisión. Provienen de circunstancias que no pueden ser apropiadamente relacionadas a los eventos actuales.

Del mismo modo, las experiencias del management contemporáneo no son tan útiles como podrían serlo para otros managers. Todavía encontramos que cada compañía y cada industria creen que sus problemas son únicos. [...] Debido a la carencia de un punto de vista fundamental adecuado, fallamos en ver cómo todas las experiencias industriales lidian con los mismos factores materiales, financieros y humanos todos representan variaciones en el mismo sistema subyacente. [...]

[...] Sin una ciencia subyacente, el avance de un arte alcanza una meseta. El management ha alcanzado tal meseta. Si el progreso va a continuar, una ciencia aplicada debe construirse como fundamento para soportar el desarrollo posterior del arte. Tal base de ciencia aplicada permitiría que las experiencias sean transmitidas a un marco de referencia común desde el cual serían transferidos desde el pasado al presente o desde una locación a otra, para ser efectivamente aplicados a nuevas situaciones por otros managers. (Forrester 2013, pp. 2-3)

Si bien Forrester reconocería luego el importante antecedente en la búsqueda de la base científica del management en la disciplina conocida como "investigación operativa" (la cual a su vez tiene por base el control estadístico de calidad de principios del siglo XX), enfatizó que ésta aún no estaba suficientemente madura en el momento que comenzó a ser el centro de las actividades de investigación del autor.

Es importante considerar también el rol de la computadora digital, cuyas infinitas aplicaciones aún no habían sido sospechadas. Esta tecnología cambiaría drásticamente las prácticas del management permitiendo encarar nuevos problemas: "Sin ella, la vasta cantidad de trabajo para obtener soluciones específicas de las características de los sistemas complejos sería prohibitivamente costosa" (Forrester 2013, p. 18). 
No hay que desdeñar, a su vez, las distintas cuestiones coyunturales que llevaron a Forrester a trabajar junto a Gordon Brown en la teoría de servomecanismos y el éxito de esa empresa (académico, pero, sobre todo, en sus aplicaciones militares), que lo llevó a dirigir como manager (pero también como ingeniero de desarrollo) un proyecto de gran envergadura: la ya mencionada computadora digital Whirlwind, la cual también terminó desempeñando un rol militar crucial. Esta experiencia como manager resultaría fundamental para lo que sucedería después (ver Giri \& Miguel 2016).

Comencemos el análisis historiográfico desde la lente epistemológica. Kuhn propuso como unidad de análisis epistemológico lo que dio en llamar en la primera edición de La estructura de las revoluciones científicas un "paradigma" y posteriormente, en su Posdata de 1969, una "matriz disciplinar" (ver Kuhn 1971). Esta unidad es más amplia que el mero conjunto de enunciados denominados "teoría" que solía representar la unidad de análisis de la Concepción Heredada (ver Suppe 1979). El concepto de matriz disciplinar (o disciplinaria) es explicado en varios textos del autor, aunque el grado de detalle es mayor en Kuhn (1982):

¿Qué elementos compartidos explican el carácter de comunicación profesional, relativamente carente de
problemas, y la unanimidad también relativa del juicio profesional? A esta pregunta La estructura de las
revoluciones científicas contesta: "un paradigma" o "un conjunto de paradigmas". [...] se produciría menos
confusión denotándolo con la frase "Matriz disciplinaria"-“disciplinaria" porque es la posesión común
de los profesionales de una disciplina y "matriz" porque se compone de elementos ordenados de distintas
maneras, cada una de las cuales hay que especificar-. Los componentes de la matriz disciplinaria incluyen
la mayoría, o todos los objetos, del compromiso de grupo descrito en el libro como paradigmas, partes de
paradigmas o paradigmático. [...] Permítaseme llamarlos generalizaciones simbólicas, modelos y
ejemplares. (Kuhn 1982, p. 321)

Como se puede apreciar de esta reseña de los episodios históricos relevantes, Forrester desarrolló gradualmente una mirada particular para enfocar problemas, partiendo de sus experiencias educativas primarias y llegando a la culminación de su aprendizaje ingenieril que incluyó una praxis concreta en aplicaciones tecnológicas varias. Es evidente que no puede reconocerse un instante preciso donde Forrester logró las ideas que lo llevarían a su novedoso enfoque, sino que éste ha sido el fruto de un largo proceso lleno de desafíos, personajes influyentes y varios hechos fortuitos.

La educación en management, por aquellos años, era criticada por varios motivos. Drucker (1959) afirmó que no se enseñaba a lidiar con los procesos de gestión del riesgo en los negocios. Forrester a su vez planteó que, al igual que en la literatura académica, en las aulas también se trabajaba sobre casuística suelta, en ausencia de un principio unificador que enseñar a los estudiantes, mientras que, por otro lado, se realizaban modelos matemáticos de tal nivel de abstracción que dificultosamente podían aportar algo útil al manager del mundo real:

La economía matemática y la [antigua] ciencia del management a menudo han sido aliados más cercanos a la matemática formal que a la economía y al management [aplicados]. La diferencia en el punto de vista es evidente si uno compara la literatura de negocios con las publicaciones en ciencia del management, o libros de economía descriptiva con textos de economía matemática. En muchos artículos de revistas profesionales la actitud es la de un ejercicio de lógica formal más que la de una búsqueda de soluciones útiles a problemas reales. En tales artículos, los presupuestos tienen dudosa validez y son fijados en un párrafo introductorio y adoptados sin justificación. En esta fundación formal pero irrealista se construye luego una solución analítica matemática para el comportamiento del sistema asumido.

Otra evidencia del sesgo de mucha de la ciencia del management actual [la antigua] hacia la motivación matemática más que gerencial es vista en una preocupación por las soluciones "óptimas". Para la mayoría de los grandes problemas del management, los métodos matemáticos son incapaces de encontrar la "mejor" solución. El objetivo confundente de buscar solo una solución óptima a menudo resulta en simplificar el problema hasta que queda vacío de interés práctico. (Forrester 2013, p. 3) 
La Dinámica de Sistemas fue el aporte de Forrester para llegar a este ansiado principio unificador con interés práctico, y así avanzar hacia un crecimiento epistémico de la ciencia del management. En términos del filósofo inglés Philip Kitcher:

La ciencia mejora nuestro entendimiento de la naturaleza mostrándonos cómo derivar descripciones de múltiples fenómenos, utilizando los mismos patrones de derivación una y otra vez [...] nos enseña cómo reducir el número de clases de hechos que tenemos que aceptar como esenciales (Kitcher 1989, p. 432).

Si bien Kitcher hacía referencia en la cita a su modelo de explicación unificacionista para las ciencias naturales, no parece haber motivos para pensar que dicho esquema de explicación científica no pueda ser aplicado también a las ciencias sociales como la que es objeto de este trabajo. Después de todo, como veremos luego, los principios de sistemas han sido aplicado exitosamente a una infinidad de campos científicos. El hecho de que tales principios (que incluyen, entre otras cosas, un tipo de lenguaje, método y esquema explicativo particular) pueda aplicarse exitosamente a una variedad de disciplinas disímiles no sólo aumenta el consenso sobre la potencia epistémica de la Dinámica de Sistemas sino que también, recíprocamente, provee mayor potencia epistémica a cada una de las disciplinas en las que se aplica, hecho especialmente relevante para el management, disciplina en la cual, como ya mencionamos, no había grandes consensos respecto a su cientificidad.

Industrial Dynamics (Forrester 2013), de 1961, fue el texto iniciático de esta nueva matriz disciplinar. Aquí el autor planteó a la Dinámica de Sistemas como aquello que aportaría finalmente el esqueleto para una ciencia progresiva del management, modelando una empresa como un "sistema" de partes interconectadas que intercambian información, dinero, órdenes, materiales, personal y equipamiento entre sí y con el ambiente exterior. Estas partes o subsistemas se denominan "sistemas de retroalimentación”, dado que sus salidas tienen influencia, ya sea de manera directa o indirecta, con sus entradas. Estos principios utilizados en la disciplina general de la cibernética, ya comenzaban incipientemente a ser aplicados para la investigación operativa y el management (ver p.e. Beer 1967) y aún constituyen el fundamento general de una rama de la disciplina de la administración industrial.

El estado de cada subsistema es descripto por su "nivel" (por ejemplo, en el caso del subsistema "depósito" su nivel es la "cantidad de stock"), y la variación del mismo es su "tasa”. Por otra parte, la reacción de cada subsistema a un input puede que no sea instantánea, sino que ocurra algún "delay" que retrase dicha reacción. Así, el funcionamiento de una empresa puede modelarse en función de los niveles de los distintos subsistemas y su variación en el tiempo, que es a su vez función de las tasas y de la estructura que interconecta cada subsistema con el resto y recibe y entrega flujos hacia el exterior. La representación puede programarse computacionalmente y a partir de allí pueden realizarse simulaciones dinámicas con el fin de definir políticas empresariales y tomar decisiones.

Un ejemplo de modelo fundamentado en la Dinámica de Sistemas que utiliza Forrester (2013) con propósitos didácticos, es el de una compañía que fabrica y distribuye un producto. Simplificadamente, se modela a dicha compañía como compuesta de tres sectores: retail (ventas), distribución y fábrica. La heurística de modelado consiste en, primeramente, identificar para cada sector las variables relevantes, es decir los niveles, tasas de flujo y delays en la circulación de información, órdenes y materiales (dinero, personal y equipos son ignorados bajo la suposición de que se hallan disponibles en cantidad suficiente como para no influir en el comportamiento del sistema). Por ejemplo, para el sector retail, los niveles son:

- Registro de órdenes recibidas del consumidor pero aún no completadas

- Inventario de producto en stock

- Promedio de ventas en el pasado reciente, utilizadas para decidir el inventario deseado y los niveles de reposición de producto 
Las tasas de flujo principales a su vez son:

- Flujo de órdenes entrantes desde los clientes

- Tasa de embarques salientes hacia los clientes

- Tasa de órdenes salientes desde el retail hacia el sector distribución

- Tasa de embarques entrantes de producto desde el sector distribución

Los delays a considerar para el caso son:

- Delay en completar las órdenes de los clientes en retail

- Delay en decidir y preparar las órdenes salientes desde retail hacia el sector distribución

- Delay en la transmisión de órdenes desde retail hacia distribución

- Delay en el despacho de producto desde distribución hacia retail

Una vez identificados estos factores, la Dinámica de Sistemas provee los elementos para realizar el modelo, plantear las ecuaciones y traducirlas al lenguaje informático DYNAMO (hoy en día en desuso, aunque existen múltiples alternativas como Vensim y Stella que operan bajo la misma lógica) a fin de realizar las simulaciones informáticas y poder tomar decisiones compatibles con los resultados obtenidos.

Forrester reconoció cuatro fundamentos centrales que habrían cristalizado en la nueva matriz disciplinar: la Teoría de Control por Retroalimentación desarrollada por su maestro Gordon Brown, que proveería la base matemática para el modelado, la Teoría de la Decisión (que si bien para la época se hallaba en estado iniciático, había producido importantes avances de la mano de la investigación operativa aplicada al ámbito militar), el enfoque experimental al análisis de sistemas (que proveería la base para la comprensión de sistemas complejos via simulaciones informáticas), y, como ya se mencionó, los avances en la tecnología informática que permitirían que las mencionadas simulaciones informáticas pudiesen representar sistemas cada vez más complejos a un costo exponencialmente menor. Debería acaso haber mencionado también los esfuerzos de los teóricos predecesores (en especial a Simon y a los cibernéticos), aunque podemos hipotetizar que probablemente no lo haya hecho a fin de enfatizar la originalidad de su propio aporte.

\section{Análisis de la matriz disciplinar}

Atendiendo a la descripción que proveyó Forrester del estado de la disciplina del management industrial, puede notarse que la ausencia de un principio unificador no permite un esfuerzo mancomunado de la comunidad científica en la búsqueda de soluciones, propio del estadio de la dinámica científica denominado por Kuhn (1971) "ciencia normal”. La descripción que provee Forrester de la disciplina señala su interpretación de la misma como inmadura, y los esfuerzos de su obra desde el MIT apuntan a proveer un marco unificador. Cabe señalar que más allá de haber logrado el objetivo de cristalizar un marco capaz de dar cuenta de los fenómenos propios de las organizaciones industriales, el mismo no reemplazó a sus predecesores, sino que se integró al conjunto de matrices disciplinares que son tenidos en cuenta por los administradores. Ahora bien, admitir que esta coexistencia de paradigmas implica necesariamente "inmadurez" significa asignarle tal carácter a todas las ciencias sociales (y también a parte de las naturales), lo que nos resulta chocante dada la proliferación de trabajos relevantes en sus distintas áreas, por lo que evitaremos tal denominación. ${ }^{2}$ Los marcos coexistentes que la Dinámica de Sistemas

\footnotetext{
2 Kuhn (2002a) en nota al pie menciona, respecto de su abandono del término "paradigma”, que:

El cambio que acabo de perfilar en mi texto me priva del recurso a expresiones como "período preparadigmático" y "período posparadigmático» cuando se describe la maduración de una especialidad científica. En retrospectiva esto me parece totalmente positivo, porque cualquier comunidad científica, incluyendo las escuelas de lo que yo llamé previamente el "período preparadigmático», ha poseído siempre paradigmas [...]. El hecho de no haber considerado este punto con anterioridad ciertamente
} 
intentó integrar eran competidores, en el mismo sentido en que la economía neoclásica y la keynesiana lo son. Los administradores, al igual que los economistas, suelen ser entrenados para poder "hablar" todos (o la mayoría) de los léxicos, aunque en última instancia elijan uno como su favorito, pero no por ello dejan de interactuar con especialistas de las otras "tribus" (en términos quineanos). Lo cierto es que más allá de la perspectiva unificadora (ingenieril-matemática) de la Dinámica de Sistemas, la misma soslaya una cantidad de elementos psicológicos individuales y sociales que hace que resulte chocante para buena parte de la comunidad de administradores, más habituados a la perspectiva "humanística" de otras escuelas de administración (como la defendida en Harvard o Chicago en la época en que Sloan funda la escuela de management en el MIT). Otro elemento soslayado por la Dinámica de Sistemas que ya mencionamos es la necesidad de ecuaciones matemáticas resolubles con resultados exactos. Al ser una perspectiva diseñada para su tratamiento por computadoras utiliza métodos numéricos inexactos, de aproximación, pues se halla interesado en predicción de tendencias generales. Esto no es una debilidad de la Dinámica de Sistemas (más bien es una fortaleza), pero de todas maneras ha provocado que no se vuelva una escuela hegemónica, aunque no pueda desdeñarse su más que considerable éxito a nivel global. Así pues, quienes la utilizan suelen complementar sus resultados con otros métodos competidores para ampliar la base de consenso en la toma de decisiones.

El management anterior a la Dinámica de Sistemas poseía su terminología propia, y la matriz disciplinar que desarrolla Jay Forrester introduciría nuevos términos, anteriormente ausentes: "retroalimentación positiva", "retroalimentación negativa", "sistema abierto", "sistema cerrado", "nivel”, "comportamiento del sistema”, "inestabilidad", "respuesta no lineal", "tasa de flujo", "delay" entre algunos otros términos que eran propios de la ingeniería (específicamente, de la Teoría de Control por Retroalimentación, aunque también adoptados por la cibernética).

Del ejemplo de la sección anterior puede verse que el término "nivel”, que no era parte del léxico anterior (en el management), ahora aprehende como clase a distintas referencias del antiguo léxico, tales como "registro de órdenes", "inventario de producto en stock", "promedio de ventas", etc. De este modo, ahora las entidades referidas por "inventario de producto en stock" (básicamente producto) y las referidas por "registro de órdenes" (básicamente órdenes de compra de los clientes) pasan a verse como entidades de un mismo tipo, esta vez, del tipo referido por "nivel". En términos kuhnianos, se podría decir que cambia la caracterización de las entidades de un mismo tipo y también cambian las relaciones de analogía.

Otros términos directamente no aprehenden ninguna referencia del management primitivo: "sistema de retroalimentación negativa de primer orden” no diría nada a un gerente de la década del '40. Es decir, estos términos nuevos ponen en evidencia la existencia de una clase de objetos que antes no se registraban como parte del objeto de estudio.

Definitivamente ha cambiado no solamente la terminología sino también los procesos, entidades o recortes a los que se refieren los términos que persisten. Así, pueden encontrarse algunos términos que persisten pero son incorporados a clases de entidades nuevas (ahora son, a los ojos de la Dinámica de Sistemas, niveles, tasas de flujo y delays), otros que son nuevos y aprehenden términos antiguos, y finalmente hay aparición de otros términos nuevos que refieren a entidades que no preexistían. Para el

ha ayudado a que el paradigma parezca una entidad cuasi-mística o una propiedad que, como el carisma, transforma a los infectados por él. Sin embargo, nótese [...] que esta alteración en la terminología no altera en absoluto mi descripción del proceso de maduración. Los primeros estadios del desarrollo de la mayoría de las ciencias se caracterizan por la presencia de cierto número de escuelas en competición. Después, generalmente como consecuencia de un logro científico notable, todas o la mayoría de las escuelas desaparecen, un cambio que permite a los miembros de la comunidad que queda una conducta profesional mucho más poderosa. (Kuhn 2002a, pp. 202-203)

Los aportes teóricos de la primera mitad del siglo XX a la disciplina parecen suficientemente significativos como para que sea injusto denominar a la disciplina como "inmadura", y refuerza el sesgo naturalista que poseen algunos sectores de la academia, incluso en el management (ver p.e. Ghoshal 2005). En tal sentido, preferiremos, en línea con Masterman (1970) la denominación del estado de la disciplina como "poliparadigmática", para señalar simplemente la existencia de una situación de competencia entre escuelas científicas rivales, como señaló un revisor anónimo aporte siguiendo a Hoyningen-Huene (2013), a quien agradezco la contribución. 
caso bajo análisis, no se registra la desaparición de términos. Finalmente, muchos términos centrales como "demanda", "tiempo" y "decisión" conservan su traducibilidad directa.

Kuhn (2002b) afirmó que:

\begin{abstract}
Afirmar que dos teorías son inconmensurables significa afirmar que no hay ningún lenguaje, neutral o de cualquier otro tipo, al que ambas teorías, concebidas como conjuntos de enunciados, puedan traducirse sin resto o pérdida. Ni en su forma metafórica ni en su forma literal inconmensurabilidad implica incomparabilidad [...]. La mayoría de los términos comunes a las dos teorías funcionan de la misma forma en ambas; sus significados, cualesquiera que puedan ser, se preservan; su traducción es simplemente homófona. Surgen problemas de traducción únicamente con un pequeño subgrupo de términos (que usualmente se interdefinen) y con los enunciados que los contienen. [...]

Llamaré «inconmensurabilidad local» a esta versión modesta de la inconmensurabilidad. (Kuhn 2002b, p. 50)
\end{abstract}

Puede decirse entonces que las modificaciones en el léxico del management introducidas por la Dinámica de Sistemas provocan inconmensurabilidad local entre la nueva matriz disciplinar y sus matrices predecesoras, principalmente debido a la introducción de nuevos términos. Esto, como explicó Kuhn, no significa bajo ningún punto de vista que el léxico nuevo y el antiguo no puedan compararse (de hecho la base lexical común, compuesta de términos persistentes, configura la estructura desde la cual la comparabilidad queda garantizada), pero la ampliación lexical genera la intraducibilidad sintomática de la inconmensurabilidad, especialmente por el agregado de términos nuevos que no pueden ser expresados en el antiguo léxico. De hecho, en la práctica la Dinámica de Sistemas se utiliza de manera complementaria a otras matrices del management, y es común que los administradores sean entrenados para entender su léxico.

Es importante para entender el modo en que el cambio teórico ha impactado en el léxico del management tener en cuenta que la base empírica de la Dinámica de Sistemas y la de sus escuelas competidoras es la misma: a saber, el comportamiento organizacional (del cual se derivan principios técnicos acerca del mejor modo de administrarlos en situaciones particulares). La particularidad de la Dinámica de Sistemas está en el nivel de abstracción de su léxico: sus términos principales (p.e. "sistema", "nivel" o "flujo") funcionan designando clases naturales que capturan entidades que existían en sus predecesoras (p.e. "empresa industrial”, "stock", "ventas", respectivamente). Las generalizaciones simbólicas, como veremos a continuación, se postulan en la Dinámica de Sistemas sobre los términos de clase natural. Forrester, como vimos, protestaba enfáticamente en su Industrial Dynamics por los modelos matemáticos de organizaciones previos a su obra, pues afirmaba que los mismos sólo poseían validez local. Traduciendo esto al lenguaje de la Dinámica de Sistemas, los modelos pre-Forrester sólo podían dar cuenta del sistema modelado, pues eran incapaces de abstraer los elementos comunes a todas las organizaciones. La clase natural "sistema", en cambio, denota cualquier organización (o sector de la organización, como retail o depósito, que son subsistemas), y las ecuaciones de la Dinámica de Sistemas valen para todo sistema, mientras que, en los modelos anteriores, cada organización y sus partes eran tan particulares que sus ecuaciones características no podían utilizarse para describir otras organizaciones (y sus partes), careciendo de generalidad.

La traducibilidad directa se da en el hecho de que, al compartirse la base empírica, muchos términos permanecen iguales, tal es la jerga de la tribu de los trabajadores de las organizaciones industriales: un depósito sigue siendo un depósito. La inconmensurabilidad se da en la ruptura del principio de nosuperposición (Kuhn 2002c), que se da en virtud de que el mismo objeto (supongamos, retail), con la Dinámica de Sistemas pasa a ser una entidad de la misma clase (y por ende sujeto a las mismas generalizaciones simbólicas) que otros objetos (por ejemplo, el depósito de materias primas) que anteriormente eran modelados con otra clase de ecuaciones en virtud de no pertenecer a la misma clase. Un caso análogo es uno de los favoritos de Kuhn, el de la revolución copernicana. Los objetos a los que refiere la astronomía aristotélico-ptolemaica y la copernicana son los mismos (Tierra, Luna, Marte, Sol, 
estrellas fijas), pero tras la revolución se rompe el principio de no superposición porque los objetos pasan a pertenecer a clases naturales diferentes. La Tierra deja de ser un objeto único en su clase (análogamente al depósito o a retail en nuestro ejemplo) para ser una entidad más en la clase natural "planeta" y estar sujeto a las mismas leyes que los demás de su clase (p.e. Marte y Venus, los cuales, junto a la Tierra, cumplen con la ley "Todos los planetas giran alrededor del Sol"). Por síntesis, dejamos al oficio de los lectores completar la analogía con este caso tan ubicuo en nuestra literatura.

Continuando con el análisis de la matriz disciplinar, se identificarán sus elementos constituyentes según Kuhn: generalizaciones simbólicas, modelos (o analogías) y ejemplares. Se agregarán a éstos también los "valores" propios de la matriz.

Las generalizaciones simbólicas "[...] son aquellas expresiones, empleadas sin cuestionamiento por el grupo, que pueden verterse fácilmente en alguna forma lógica como $(x)(y)(z) \phi(x, y, z)$. Son los componentes formales, o fáciles de formalizar, de la matriz disciplinaria” (Kuhn 1982, p. 321).

En Forrester (2013) se dedicó mucho espacio a mostrar las ecuaciones matemáticas (derivadas de la Teoría de Control por Retroalimentación) a través de las cuales pueden modelarse las distintas situaciones del management de las cuales da cuenta la nueva matriz. Como ejemplo, se volverá a traer a colación el caso del sector retail. Uno de los niveles identificados para el sector era el "registro de órdenes recibidas del consumidor pero aún no completadas" (UOR), mientras otro era el "inventario de producto en stock" (IAR). Ambos niveles son de los más sencillos en los modelos de la Dinámica de Sistemas, puesto que en ambos casos, existe un único flujo de entrada (de órdenes en UOR, y de producto en IAR) y uno sólo de salida (de lo mismo). Las ecuaciones estándar de esta matriz disciplinar, para este tipo de niveles, se instancia para UOR de la siguiente manera:

$$
\begin{aligned}
\text { UOR.K } & =\text { UOR.J + (DT) (RRR.JK }- \text { SSR.JK) } \\
\text { UOR } & =\text { órdenes incompletas en retail (medido en unidades de producto en las órdenes) } \\
\text { RRR } & =\text { requisiciones (órdenes) recibidas en retail (unidades de producto por semana) } \\
\text { SSR } & =\text { despachos enviados desde retail (unidades de producto por semana) } \\
\text { DT } & =\text { delta de tiempo (semanas), es decir, el intervalo de tiempo entre las soluciones de } \\
& \text { las ecuaciones. }
\end{aligned}
$$

La ecuación calcula el número de órdenes incompletas en el tiempo presente $\mathrm{K}$, basada en las órdenes incompletas calculadas en el tiempo anterior J, y en las tasas de flujo de entrada y salida durante el intervalo de tiempo JK entre cálculos de UOR. Las tasas de flujo de entrada y salida son asumidas constantes durante el intervalo JK [...]. El largo del intervalo de tiempo DT multiplicado por la tasa de flujo de entrada RRR.JK provee las nuevas órdenes recibidas en el intervalo JK. Del mismo modo, (DT)(SSR.JK) provee las órdenes completadas en el intervalo JK. (Forrester 2013 p. 142)

La ecuación de un nivel es, en términos más sencillos, lo que en ingeniería se denomina un "balance de masa". Si quiere saberse qué volumen de agua posee un tanque en todo momento, uno debe saber lo que tenía en el intervalo de tiempo anterior, sumarle el agua que entró y restarle la que salió del tanque. El mismo razonamiento podría realizarse para sistemas de otro tipo, por ejemplo, para monitorear la cantidad de dinero que posee en todo momento una cuenta bancaria. Este tipo de analogías son constitutivas de la matriz disciplinar que estamos analizando.

Por supuesto, existen niveles más complejos que el del ejemplo, y hay ecuaciones para dar cuenta de ellos, de las tasas de flujo y de los delays. Las relaciones entre esos componentes, ya sea que se hallen formalizados o no, constituyen entonces las distintas leyes de la Dinámica de Sistemas La generalización simbólica de la Dinámica de Sistemas puede señalarse con la siguiente ecuación, que describe el nivel de cualquier sistema en un instante $\mathrm{K}$ :

$$
\begin{aligned}
& \text { N.K }=\text { N.J + (DT) }(\text { RR.JK }- \text { SS.JK) } \\
& \text { N.K }=\text { Nivel del sistema en el momento } K
\end{aligned}
$$


N.J = Nivel del sistema en el momento $\mathrm{J}$ (donde $\mathrm{J}$ es el instante temporal anterior a $\mathrm{K}$ )

RR.JK = Valor deseado de salida entre $\mathrm{J}$ y $\mathrm{K}$

SS. JK = Valor real de salida entre J y K

$\mathrm{DT}=$ Salto de tiempo entre J y K

Puede verse que la ecuación para el sistema retail presentada arriba como ejemplo es una instanciación de esta generalización simbólica.

En cuanto a los modelos, Kuhn afirmó que:

[...] proveen al grupo de analogías preferentes o, cuando se sostienen profundamente, de una ontología. Por una parte, son heurísticos: el circuito eléctrico puede considerarse, provechosamente, como un sistema hidrodinámico en estado estable, o el comportamiento de un gas, como el de una colección de microscópicas bolas de billar en movimiento aleatorio. Por otra parte, son los objetos del compromiso metafísico: el calor de un cuerpo es la energía cinética de sus partículas componentes, o, más obviamente metafísico, todos los fenómenos perceptibles se deben al movimiento y a la interacción de átomos cualitativamente neutrales, en el vacío. (Kuhn 1982, pp. 321-322)

Los modelos de esta matriz disciplinar son aquellas analogías surgidas de la exportación de ontologías sistémicas de la ingeniería hacia el management: modeladas bajo el crisol de la Dinámica de Sistemas, las compañías no serían distintas (y por ende les corresponderían las mismas generalizaciones simbólicas) que las máquinas electrohidráulicas controladas mediante mecanismos de retroalimentación que constituían los ejemplares exitosos de la Teoría de Servomecanismos de Gordon Brown. Por otra parte, la Teoría de la Decisión surgida desde el seno militar, vista desde cierto nivel de abstracción, resulta, desde la nueva matriz disciplinar del management, perfectamente compatible con una metodología adecuada para el diseño de políticas decisorias en el ámbito empresarial (buena parte de los desarrollos del management surgieron primero desde el sector militar). Estos compromisos proveen heurísticas que permiten resolver una variedad de problemas que el viejo management no podía abordar sistemáticamente, y que instancian la gran novedad de la matriz disciplinar creada por Jay Forrester.

Los valores fueron descriptos en la Posdata de 1969 como otro de los elementos fundamentales de las matrices disciplinares:

Habitualmente se les comparte entre diferentes comunidades, más generalmente que las generalizaciones simbólicas o los modelos, y hacen mucho para dar un sentido de comunidad a los científicos naturalistas en conjunto. Aunque funcionan en todo momento, su importancia particular surge cuando los miembros de una comunidad particular deben identificar una crisis o, después, escoger entre formas incompatibles de practicar su disciplina. Probablemente los valores más profundamente sostenidos se refieren a las predicciones: deben ser exactas; las predicciones cuantitativas son preferibles a las cualitativas; sea cual fuere el margen de error admisible, debe ser continuamente respetado en un campo determinado, y así por el estilo. (Kuhn 1971, p. 283)

La pretensión de obtener "predicciones exactas" no es compatible con la mayoría de las ciencias que abordan sistemas complejos como los del management (y la mayor parte de las ciencias sociales). Sin embargo, la nueva matriz disciplinar ha conseguido proveer tendencias de comportamiento de los sistemas lo suficientemente útiles como para tomar decisiones correctas y formular políticas que permitan mejorar el diseño del sistema bajo estudio:

Debería entonces esperarse de un modelo de un sistema dinámico que represente y prediga las características de comportamiento (como la rentabilidad, estabilidad de empleo y precios, tendencia de crecimiento, y relaciones periódicas típicas de cambios en las variables) del sistema presente. No debería esperarse que prediga estados futuros del sistema excepto en la medida que el sistema tenga continuidad y características de momentum que causen que las condiciones presentes y las tendencias persistan por un tiempo a pesar de las perturbaciones del ruido (Forrester 2013, p. 125). 
Puede verse que la matriz disciplinar se ha alejado de las predicciones cuantitativas exactas para acercarse a predicciones de comportamiento útiles para la mejora de los sistemas. Así, la Dinámica de Sistemas cambió su apreciación de las predicciones respecto al antiguo management, que, como se vio en secciones anteriores, privilegiaba modelos cuantitativos analíticamente resolubles cuyas soluciones óptimas exactas no arrojaban ningún conocimiento útil aplicable a sistemas del mundo real. Este tipo de modelos, matemáticamente elegantes pero pragmáticamente inútiles, deben abandonarse según la nueva matriz (ciertamente no lo hicieron, ni llevaron al abandono de la gran mayoría de los principios teóricos del management clásico). El valor supremo desde la visión de Forrester se encontraría en la resolución aproximada de problemas de compañías reales. No resulta casual que esta visión instrumental, ubicua en la racionalidad ingenieril, haya llegado al management de la mano de un ingeniero.

Otro de los elementos característicos de las matrices disciplinares, según Kuhn, son sus "ejemplares”, los cuales "[...] son soluciones de problemas concretos aceptadas por el grupo [la comunidad científica] como paradigmáticas en el sentido usual del término" (1982, p. 322). En otras palabras, el ejemplar es todo aquel caso de aplicación de la matriz disciplinar que resulta exitoso, y buena cantidad de éstos toman la forma de ejercicios a resolver en los manuales de enseñanza científica.

Ciertamente, la Dinámica de Sistemas fue utilizado en áreas disciplinares realmente disímiles, como por ejemplo la educación (p.e. Groff 2013) o la biología (p.e. Yin \& Struick 2010), pero dentro del management son especialmente notables las aplicaciones a la gestión urbana en Urban Dynamics (Forrester 1999) o el famoso y polémico World Dynamics (Forrester 1973), el cual fue el antecesor del aún más famoso y (mucho más) polémico Limits to Growth (Meadows et al. 1972), texto auspiciado por el Club de Roma que llevó a la cristalización de la agenda ecologista en el mundo, al proponer la imposibilidad del crecimiento económico infinito en un mundo finito (ver Bardi 2011, Giri 2017).

Finalmente, destacamos la agrupación institucional de los científicos que trabajan bajo esta matriz disciplinar en la System Dynamics Society, fundada en 1982. Hoy en día la Dinámica de Sistemas constituye el marco de base de un importante grupo de científicos de la administración (sobre todo de su vertiente industrial).0

\section{Conclusiones}

De la descripción histórica presentada pueden observarse los siguientes puntos destacables.

En 1957 confluyeron una serie de factores que proveerian un marco propicio para lo que fue identificado en este capítulo como la creación de una matriz disciplinar del management. Esta permitiría el surgimiento de nuevas formas de concebir la composición de los sistemas en estudio, esto es, las empresas industriales, las ciudades, el mundo y sus dinámicas.

Por una parte, existía una ciencia del management que planteaba preguntas pero otorgaba respuestas insatisfactorias (constituyendo anomalías) y provocaba que los científicos y las compañías demandasen nuevas soluciones.

En segundo lugar, puede notarse una Teoría matemática de Control por Retroalimentación que, en combinación con la Teoría de la Decisión derivada del ámbito militar, fue capaz de modelar sistemas como los que son objetos de estudio del management, aunque hasta ese momento sólo había sido utilizada por la ingeniería en automatización de procesos electromecánicos.

En tercer lugar, se identifica una ciencia informática, incipiente en el período estudiado, que permitió formalizar los modelos de la Teoría de Control por Retroalimentación para llevarla a lenguaje de máquina. De este modo se podrían realizar simulaciones computacionales para plantear y estudiar escenarios variados constituyendo así una herramienta útil para la toma de decisiones.

En cuarto lugar, se señaló la aparición de una tecnología nueva, la computadora digital, que proveyó las condiciones de posibilidad para realizar dichas simulaciones. 
Los factores señalados hasta aquí cristalizaron en una nueva matriz disciplinar de la ciencia del management industrial, la Dinámica de Sistemas, la cual posee todos los ingredientes mencionados por Kuhn para ese tipo de unidad epistemológica de análisis:

a) Hubo cambios en las generalizaciones simbólicas (pasaron a agregarse aquellas formalizaciones de la Teoría matemática del Control por Retroalimentación).

b) Aparecieron cambios en los modelos (las compañías comenzaron a ser análogas a máquinas con módulos de control automático por retroalimentación).

c) Se modificaron los valores (las descripciones sueltas de la casuística del antiguo management y los modelos matemáticos abstractos de escasa utilidad práctica fueron abandonados (dentro de la matriz, aunque persistieron en el management general) por modelos que no buscan predicciones cuantitativamente precisas, sino más bien, proveer pautas y señales para el diseño de mejores sistemas industriales en el mundo real).

d) Llegaron nuevos y progresivos ejemplares.

También se describió la introducción de una nueva terminología, de modo tal que la nueva matriz disciplinar posee inconmensurabilidad local con las distintas concepciones del management de la etapa predecesora.

Los primeros ejemplares exitosos de esta teoría fueron desarrollados para el management industrial (Industrial Dynamics), pero el planteo de enigmas diferentes llevó a extender el método tanto a la administración de ciudades (Urban Dynamics), como a la administración de la economía del mundo como un todo (World Dynamics).

La matriz disciplinar "Dinámica de Sistemas" actualmente está vigente y sigue siendo fructífera. En el año 1982 apareció una institución internacional que hoy sigue nucleando a la comunidad científica que trabaja bajo esta matriz: la System Dynamics Society. Los valores pregonados por esta organización son compartidos por sus miembros qua científicos enmarcados en una matriz disciplinar, todo lo cual puede identificarse con la constitución de una comunidad científica (o tribu, en la analogía antropológica típica de Quine 2013) alrededor de estas prácticas, que sustentan la misma manera de concebir los sistemas objeto de estudio tanto en sus componentes como en el estilo de abordar su dinámica. En otras palabras, comparten generalizaciones simbólicas, modelos, valores y ejemplares.

Puede concluirse también que los detalles del cambio conceptual introducidos por Jay Wright Forrester al management han sido capturados con un grado de precisión interesante por la metateoría kuhniana, siendo esta propicia a tal fin, aunque deba precisarse que lo que hemos ilustrado no constituye la formación de un paradigma único que desplaza a sus competidores y logra la cohesión total de la comunidad, como sucede en los ejemplos emblemáticos de la revolución copernicana o la revolución química que ilustra Kuhn (1971). Lo que sí hemos ilustrado es la formación de una matriz disciplinar nueva que coexiste, del modo sugerido por Kuhn en su posdata, con sus matrices precedentes (y con otras posteriores), aunque es parcialmente inconmensurable con ellas. Ciertamente, el management persiste en estado poliparadigmático en nuestros días, aunque no por ello deja de ser una ciencia (aunque parte de su acervo es más bien tecnológico, ver Bunge 2012, Giri 2017) de carácter progresivo.

Bibliografía

Bardi, U. (2011), The Limits to Growth Revisited, New York: Springer.

Barnard, C. (1938), The Function of the Executive, Cambridge: Harvard University Press.

Beer, S. (1967), Cybernetics and Management, London: English Universities Press. 
Bunge, M. (2012), "Status epistemológico de la administración”, en Bunge, M., Filosofía de la tecnología y otros ensayos, Lima: Universidad Inca Garcilaso de la Vega, pp. 79-86.

Drucker, P. (1954), The Practice of Management: A Study of the Most Important Function in American Society, New York: Harper \& Brothers.

Drucker, P. (1959), “Thinking Ahead: Potentials of Management Science”, Harvard Business Review 37(1): 25-30.

Forrester, J. (1973), World Dynamics, Cambridge: Wright Allen Press.

Forrester, J. (1989), The Beginning of System Dynamics, Stuttgart: System Dynamics Society.

Forrester, J. (1992), "From the Ranch to System Dynamics: An Autobiography", en Bedeian, A. (ed.), Management Laureates: A Collection of Autobiographical Essays, vol. 1, Greenwich: JAI Press.

Forrester, J. (1995), “The Beginning of System Dynamics”, The McKinsey Quarterly 4: 4-16.

Forrester, J. (1999), Urban Dynamics, Cambridge: Wright Allen Press.

Forrester, J. (2013), Industrial Dynamics, Mansfield Centre, CT: Martino.

Ghoshal, S. (2005), “Bad Management Theories are Destroying Good Management Practices”, Academy of Management Learning $\mathcal{E}$ Education 4(1): 75-91.

Giri, L. (2017), "Modelización, predicción y valores sociales”, en Tula Molina, F. y H.G. Giuliano (eds.), El riesgo de que todo funcione: para una evaluación amplia de la tecnología. Buenos Aires: Nueva Librería, pp. 37-60.

Giri, L. y M. Giri (2020), "Recuperando un programa kuhniano en historia de la ciencia”, Cuadernos de filosofía 38: 75 89.

Giri, L. y H. Miguel (2016), “Un caso de cambio revolucionario en ciencias sociales”, en Melogno, P. (ed.), Perspectivas sobre el lenguaje científico, Montevideo: FIC-UDELAR, pp. 71-82.

Gordon, R. y J. Howell (1959), “Higher Education for Business”, The Journal of Business Education 35(3): 115-117.

Groff, J. (2013), “Dynamic Systems Modeling in Educational System Design \& Policy”, New Approaches in Educational Research 2(2): 72-81.

Gulick, L. y Urwick, L. (1937), Papers on the Science of Administration, New York: Institute of Public Administration.

Hoyningen-Huene, P. (1993), Reconstructing Scientific Revolutions: Thomas S. Kuhn's Philosophy of Science. Chicago and London: Chicago University Press.

Kitcher, P. (1989), "Explanatory Unification and the Causal Structure of the World”, en Kitcher, P. y Salmon, W. (eds.) XIII Minnesota Studies in the Philosophy of Science, Minneapolis: University of Minnesota Press, pp. 410-505.

Kochan, T. y R. Schmalensee (2003), Management: Inventing and Delivering its Future, Cambridge: The MIT Press.

Kuhn, T. (1971), La estructura de las revoluciones científicas, México: Fondo de Cultura Económica.

Kuhn, T. (1982), “Algo más sobre los paradigmas”, en Kuhn, T., La tensión esencial: estudios selectos sobre la tradición y el cambio en el ámbito de la ciencia, Madrid: Fondo de Cultura Económica, pp. 317-343.

Kuhn, T. (2017), Desarrollo científico y cambio de léxico, Montevideo: FIC-UDELAR/ANII/SADAF.

Kuhn, T. (2002a), “Consideraciones en torno a mis críticos”, en Conant, J. y J. Haugeland (eds.), El camino desde La Estructura, Buenos Aires: Paidós, pp. 151-210.

Kuhn, T. (2002b), “Conmensurabilidad, comparabilidad y comunicabilidad”, en Conant, J. y J. Haugeland (eds.), El camino desde La Estructura, Buenos Aires: Paidós, pp. 47-76.

Kuhn, T. (2002c), "El camino desde La Estructura”, en Conant, J. y J. Haugeland (eds.), El camino desde La Estructura, Buenos Aires: Paidós, pp. 113-129.

Lane, D. y J. Sterman (2011), “Jay Wright Forrester”, en Assad, A. y S. Gass (eds.), Operations Research: Pioneers and Innovators, New York: Springer, pp. 363-386. 
Masterman, M. (1970), “The Nature of a Paradigm”, en Lakatos, I. y A. Musgrave (eds.), Criticism and the Growth of Knowledge, London/New York: Cambridge University Press, pp. 59-91.

Meadows, D., Meadows, D. H., Randers, J. y W. Behrens (1972), Limits to Growth, New York: Universe Books.

Moulines, C. U. (1983), "On How the Distinction between History and Philosophy of Science Should Not Be Drawn”, Erkenntnis 19(1): 285-296.

Moulines, C. U. (2011), “Cuatro tipos de desarrollo teórico en las ciencias empíricas”, Metatheoria 1(2): 11-27.

Quine, W. (2013), Word and Object, Cambridge: MIT Press.

Simon, H. (1964), El comportamiento administrativo, Madrid: Aguilar.

Suppe, F. (1979), La estructura de las teorías científicas, Madrid: Editora Nacional.

Taylor, F. (1919), The Principles of Scientific Management, New York: Harper \& Brothers.

Von Neumann, J. y O. Morgenstern (1990), Theory of Games and Economic Behaviour, Princeton: Princeton University Press.

Weber, M. (1924), The Theory of Social and Economic Organization, New York: Oxford University Press.

Yin, X. y P. Struick (2010), "Modelling the Crop: from System Dynamics to Systems Biology", Journal of Experimental Botany 61(8): 171-2183. 